Document downloaded from:

http://hdl.handle.net/10251/44424

This paper must be cited as:

Heras Barberá, SM.; Botti Navarro, VJ.; Julian Inglada, VJ. (2009). Challenges for a CBR framework for argumentation in open MAS. Knowledge Engineering Review. 24(4):327352. doi:10.1017/S0269888909990178.

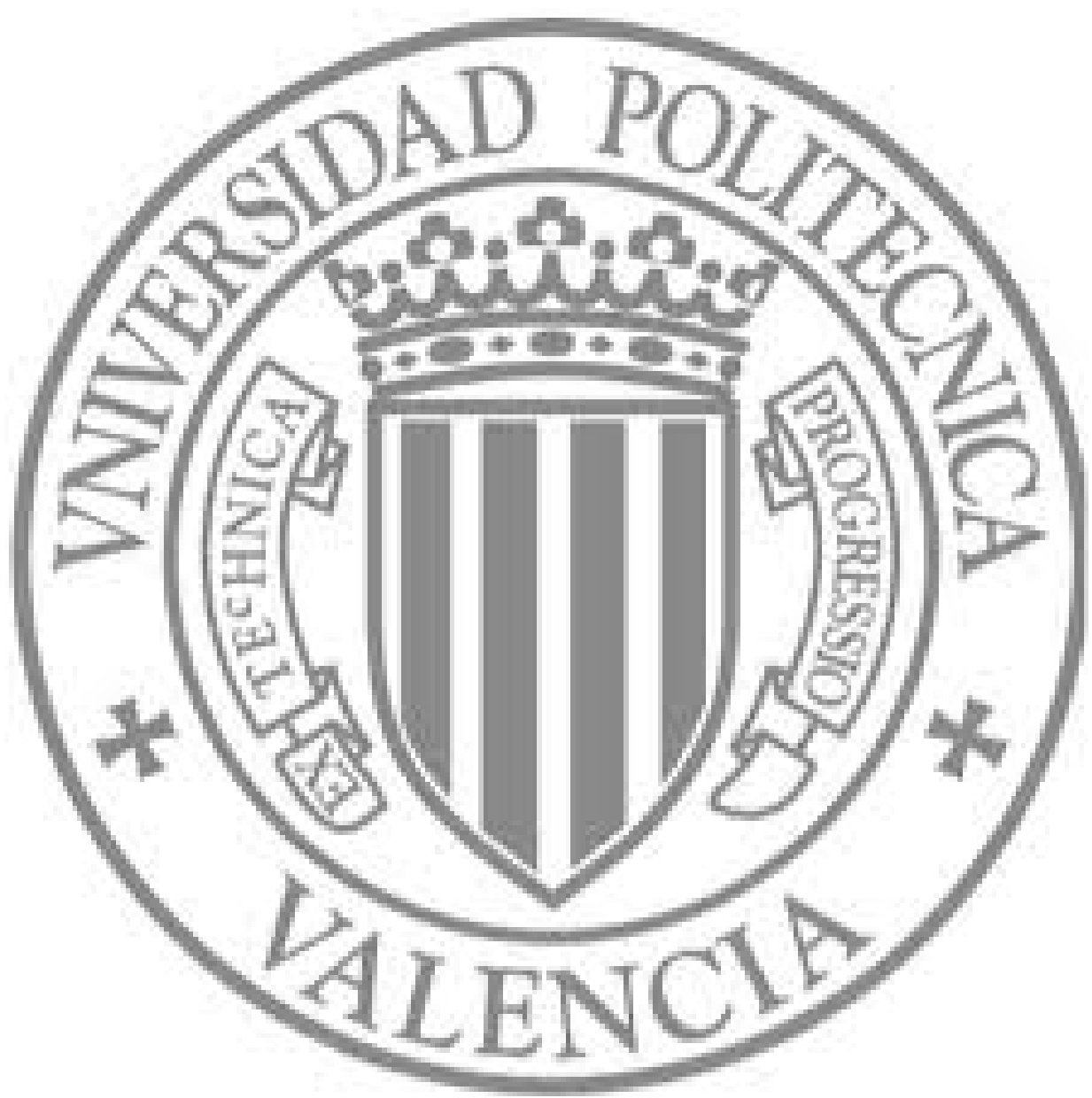

The final publication is available at

http://dx.doi.og/10.1017/S0269888909990178

Copyright

Cambridge University Press (CUP) 


\title{
Challenges for a CBR Framework for Argumentation in open MAS
}

\author{
Stella Heras, Vicente Botti \& Vicente Julián \\ Information Systems and Computing Department, \\ Universidad Politécnica de Valencia, \\ Camino de Vera s/n. 46022 Valencia, Spain \\ (+34) 963877350 Ext: 83528 \\ E-mail: \{sheras,vbotti,vinglada\}@dsic.upv.es
}

\begin{abstract}
Nowadays, Multi-Agent Systems (MAS) are broadening their applications to open environments, where heterogeneous agents could enter into the system, form agents' organisations and interact. The high dynamism of open MAS gives rise to potential conflicts between agents and thus, to a need for a mechanism to reach agreements. Argumentation is a natural way of harmonising conflicts of opinion that has been applied to many disciplines, such as Case-Based Reasoning (CBR) and MAS. Some approaches that apply CBR to manage argumentation in MAS have been proposed in the literature. These improve agents' argumentation skills by allowing them to reason and learn from experiences. In this paper, we have reviewed these approaches and identified the current contributions of the CBR methodology in this area. As a result of this work, we have proposed several open issues that must be taken into consideration to develop a CBR framework that provides the agents of an open MAS with arguing and learning capabilities.
\end{abstract}

\section{Introduction}

All along the history, research done on argumentation and on Artificial Intelligence (AI) have experienced mutual contributions. The argumentation theory has produced important benefits on many AI research areas, from its first uses as an alternative to formal logic for reasoning with incomplete and uncertain information to its more recent applications in Multi-Agent Systems (MAS) (Bench-Capon \& Dunne, 2007). Currently, the study of argumentation in this area has gained a growing interest. The reason behind is that having argumentation skills increases the agents' autonomy and provides them with a more intelligent behaviour.

An autonomous agent should be able to act and reason as an individual entity on the basis of its mental state (beliefs, desires, intentions, goals, etc.). As member of a MAS, an agent interacts with other agents whose goals could come into conflict with those of the agent. Moreover, if a dynamic and open MAS is considered, the knowledge that an agent has about the environment, its neighbours and its mental state can change in the course of time. Therefore, agents must have the ability of reaching agreements that harmonise their mental states and that solve their conflicts with other agents. Argumentation is a natural way of reaching agreements between several parties with opposing positions about a particular issue. The argumentation techniques, hence, can be used to facilitate the agents' autonomous reasoning and to specify interaction protocols between them (Rahwan, 2006).

Case-Based Reasoning (CBR) (Aamodt \& Plaza, 1994) is another research area where the argumentation theory has produced a wide history of successful applications. According to the CBR methodology, a new problem can be solved by searching in a case-base for similar precedents and adapting their solutions to fit the current problem. This reasoning methodology has a high resemblance with the way by which people argue about their positions, trying to justify them on the basis of past experiences. The 
argumentation theory concepts and techniques have been successfully applied in a great number of CBR systems, specially in those that work in legal domains, where a plaintiff and a defendant argue over their opposing positions in court. In this case, if 'common law' is the legal system that applies, similar cases should be resolved with similar verdicts.

The work done in the eighties about legal CBR fostered the argumentation research in the AI community (Rissland et al., 2006). From then on, the good results of CBR systems in argumentation domains suggest that this type of reasoning is suitable to manage argumentation processes. Nowadays, MAS research community is endeavouring to broaden the applications of the paradigm to more real environments, where heterogeneous agents could enter in (or leave) the system, form societies and interact with other agents (Ossowski \& et al., 2007). As it was pointed out before, the high dynamism of these open $M A S$ gives rise to a greater need for a way of reaching and managing agreements that harmonise conflicts. Moreover, this type of systems also poses other potential problems to overcome. Common assumptions about the agents of most MAS, such as honesty, cooperativeness and trustworthiness cannot be longer taken as valid hypothesis in open MAS. Therefore, there is an obvious need for providing the agents of an open MAS with individual reasoning and learning capabilities that make them more intelligent and autonomous and prevent them from the potential attacks of interested agents.

Our aim is to study the feasibility of using CBR as the main reasoning and learning method in a framework that allows the agents of an open MAS to reach agreements via argumentation and to learn by experience to perform a more effective (strategic) dialogue. With this purpose, we have reviewed the approaches of hybrid case-based MAS for argumentation that have been proposed in the literature. As a result of this work, the current CBR contributions to argumentation in MAS have been identified in this article. In addition, future work to perform has been specified by pointing out several open research issues that must be taken into account to develop a case-based argumentation framework for open MAS.

The present paper shows the conclusions that we have drawn, following this structure: section 2 introduces important concepts of the argumentation theory that have been adapted to argue in AI systems and also makes a review of the argumentation study in this field, section 3 analyses the contributions of CBR to argue in MAS and summarises the conclusions of this analysis and finally, section 4 proposes open issues for the application of CBR to manage argumentation dialogues in open MAS.

\section{Background}

Argumentation is a multi-disciplinary field of study that gathers contributions of many different research areas, such as philosophy, law and AI. In this section, we show the main definitions and concepts of argumentation theory that have been adopted in AI. We also make a brief review of the main applications that argumentation has had in this area, emphasising the research that has been done in CBR and MAS.

\subsection{Argumentation Theory}

Argumentation theory provides a framework to model such dialogical situations where a set of participants, which have opposing opinions about a certain claim, engage in a dialogue by generating arguments that support or attack this main claim. From its origins on the classic philosophy to nowadays, the argumentation study has given rise to many argumentation theories. A theory for argumentation specifies the elements that define the argumentation dialogue; such as, for example, the argument components, the argumentation logic, the inference rules and the argumentation protocol. Currently, one of the most accepted argumentation theories is the pragma-dialectical, proposed by Van Eemeren \& Grootendorst (2004). According to these authors:

'...Argumentation is a verbal, social, and rational activity aimed at convincing a reasonable critic of the acceptability of a standpoint by putting forward a constellation of propositions justifying or refuting the proposition expressed in the standpoint...'

Thus, justifying one's own opinion or rebutting others' is the main objective of argumentation. The argumentation process takes place over the proposition of a sequence of statements in favour 
of a claim (proarguments) or against it (counterarguments). Argumentation is aimed to increment (or decrement) the acceptability of a controversial point of view, in other words, to persuade the opponent (but in some situations collaborative decision making and negotiation are also possible objectives of the argumentation). Following van Eemeren and Grootendorst's point of view, the argumentation is part of a critical discussion. Its objective is to resolve opinion disagreements over a process that has the following stages:

- Confrontation: where the problem is presented.

- Opening: where the valid rules are determined (how to present evidences, which sources of facts are valid, how to evaluate differing opinions, termination rules, etc.).

- Argumentation: where the logical principles are applied in accordance with the rules that have been established and each party defends its position or attacks other positions.

- Concluding: where the parties reach an agreement (or do not) and the process ends.

Many MAS where the interaction between the agents is defined via an argumentation process have characteristics of the pragma-dialectical theory and, more or less explicitly, show regard for its stages in the dialogue between the agents. In addition, several well-known concepts of the argumentation theory have been adopted for the AI community to manage the argumentation between the agents of a MAS.

On one hand, the dialogue typology established by the philosophers Walton \& Krabbe (1995) has been commonly used to classify the dialogue between the agents of a MAS in view of its objective and the agents' aims. The types of dialogues that were considered in this typology are: persuasion, negotiation, inquiry, deliberation, information seeking, eristics and mixtures between them.

On the other hand, the concepts and theory of dialogue games have been adopted as a tool for specifying communication protocols between the agents of MAS (as will be shown sections below). In CBR, dialogue games have also been applied to model the human reasoning about legal precedents (Prakken \& Sartor, 1998). Dialogue games are interactions between two or more players, where each player 'moves' by posing statements in accordance with a set or predefined rules. According to the work of McBurney and Parsons (McBurney \& Parsons, 2002), the main components of dialogue games are commencement rules, locutions, combination rules, commitments and termination rules. Here, commitments are defined as rules that show the circumstances under which participants express commitment to a proposition. The concept of commitment stores has been broadly used in MAS. Such concept comes from the Hamblin's study of fallacies (Hamblin, 1970) (invalid reasoning patterns that are flawed in their logic or form and try to imitate valid reasoning patterns). This work spread the idea that formal reasoning systems should have public commitment stores for every participant of a dialogue, whose commitments can be withdrawn in view of certain circumstances. Correspondingly, the adding of a new commitment entails a previous checking to keep the coherence of the store information.

Finally, as will be shown below, argumentation schemes, proposed in argumentation theory to represent stereotyped patterns of everyday reasoning, have been adopted in MAS to model the underlying reasoning of an argumentation dialogue between agents. In these schemes, the arguments take the form of a set of inference rules by which, given a set of premises, a conclusion can be inferred. Some famous argumentation schemes are those of Toulmin (1958), Perelman \& Olbrechts-Tyteca (1969) and van Eemeren \& Grootendorst (1984). However, the argumentation schemes submitted by Walton (1996), which include a set of critical questions, are the most widely used in MAS. These questions provide a clear way for the opponent to attack the claim that the scheme supports. This is a useful feature that clarifies the structure of the dialogue, since the set of instantiations of the critical questions represents the set of possible attacks to the main claim of the argumentation scheme.

\subsection{Argumentation in AI}

From the beginning of AI research, the development of knowledge-bases, reasoning methods and cognitive architectures able to reply human reasoning has been a core area of interest. The work done in such area is typically known as common sense reasoning research. A reasoning method of this kind must include the following features: 
- The ability to manage uncertain knowledge.

- The ability to reason with knowledge that is assumed to be true or false in the absence of any evidence that shows the opposite, which is called default reasoning.

- The ability to reason quickly over a wide range of domains.

- The ability to reason and take decisions in presence of incomplete knowledge and subsequently, to revise the beliefs and decisions that were taken when concrete knowledge is acquired, which is called non-monotonic reasoning.

Initially, argumentation theory was adopted in AI due to the inability of the classic propositional logic to reason and give explanations in presence of uncertain or imprecise information (Reiter, 1980). The main problem with classical logic is its monotonic condition, which implies that the acquisition of new information cannot modify the conclusions that were inferred to that moment and thus, it is not applicable as common sense reasoning method. This problem already appeared in rule-based expert systems, where several rules could conflict or even be invalidated by the acquisition of new information. The process of drawing conclusions by using rules that can be defeated by new information is called defeasible reasoning. When defeasible rules are linked up to reach to a conclusion, the proofs that support such rules turn into arguments. The arguments can defeat each others, given rise to an argumentation process. To determine the winning arguments, they must be compared by establishing which beliefs are justified. Therefore, argumentation theory has been studied in AI to deal with the process of argument searching and more concretely (Bench-Capon \& Dunne, 2007):

- To define the argument components and their interaction.

- To identify the protocols and rules that manage the argumentation processes.

- To distinguish between valid and invalid arguments.

- To determine the conditions under which further discussion becomes redundant.

Bench-Capon and Dunne (Bench-Capon \& Dunne, 2007) provides an extensive review of the argumentation research that has been done in AI throughout the history. According to the authors, the foundations of argumentation in AI lie in the studies done to extend non-classical logic to manage argumentation, the argumentation models that are based on dialogue processes and the diagrammatic treatments of the argument structure. We refer the reader to this important survey for further details.

However, among the work that promoted the use of argumentation in AI, the research done in legal reasoning really stands out. In fact, the results obtained in the eighties from the work performed by the CBR researchers on applying this methodology of reasoning to legal domains fostered the study of argumentation in the AI community. Law is one of the most important application domains of the argumentation theory. In this domain, a case represents a conflict between two parties that argue about their opposing positions in court. Each party tries to persuade the other to obtain a verdict that was favourable to its lawsuit. The concepts that characterise legal cases cannot be universally defined by valid and sufficient conditions, but they are open-textured. Each case interpretation is arguable and hence, experts are in disagreement about what its verdict should be. An AI system suitable for working with legal cases must be able to store and manage uncertain and incomplete information.

CBR fits these conditions perfectly. Moreover, the CBR methodology has a high resemblance with the way in which people argue about their experiences. Therefore, CBR has been successfully applied in many legal reasoning systems that are based on precedents. This is the case of Anglo-American law, which follows a common law legal system whose judicial standard, stare decisis, orders that similar cases must be resolved with similar verdicts. In this domain, CBR is the most common reasoning mode. However, the judicial standard does not specify how to measure the similarity between cases. In fact, the similarity is not static, but it depends on each person's point of view and objectives. Rissland et al. (2003) report this and other features that make the legal domain an excellent area to study the CBR typical research issues (indexing, retrieval, similarity measurement, etc.).

In 1991, the seminal works of the CBR researchers Ashley, Branting, Rissland and Skalak were published. The ideas that were spread on their projects established the basis of what is known today as interpretive CBR. The first and probably the most important interpretive CBR system of that time 
was Ashley's HYPO system (Ashley, 1991). HYPO generates legal arguments by citing previous cases (precedents) as justifications of the conclusions about who should win a dispute in the domain of the trade secrets American law. Concretely, the system generates 3-ply arguments that show the best cases to cite for each party, their distinctions (the differences between the current and the previous case) and their counterexamples (previous cases that are similar to the current case and that were resolved in the other party's favour).

The main contributions of HYPO were the definition of several dimensions, in terms of which arguing in law is common, and the design of methods to compare and contrast cases on the basis of their applicable dimensions. In HYPO, the most similar cases (most on-point cases) are those that share a major number of dimensions. Therefore, the disputes in HYPO are represented by their dimensions, in view of the lack of a sound domain theory that prescribes rules for taking decisions over any legal problem. The dimensions show a set of facts that reinforce or weaken the argument of a plaintiff for a certain conclusion. However, legal experts do not reach an agreement about the relative importance of the dimensions. HYPO, thus, helps in the resolution of a new legal dispute by relating it with previous cases and by generating the whole argumention process that might be followed. In addition to this, HYPO also offers a list of hypothesis (or hypos) that modify the problem by reinforcing or weakening the position of each party. These hypos may be very useful for the plaintiff or the defendant when preparing themselves for dealing with the potential arguments that the other party might generate against his position during the trial.

The HYPO's success gave rise to the subsequent development of several systems that share its problem analysing style. The most direct descendent of HYPO and probably the most elaborated is Aleven's CATO system (Aleven \& Ashley, 1997), an intelligent learning environment that teaches law students to build arguments from cases. In addition to the typical functionalities of argumentation systems, CATO also includes other abilities that legal experts have and that law students must learn: such as to organise a written argument by topics using multiple cases and to generate arguments about the similarity between cases. To perform that, CATO extended the argumentation model of HYPO with a factor hierarchy. This hierarchy is a representation of the domain normative knowledge about the meaning of the case factors that experts have. CATO's factors are a simplification of HYPO's dimensions and represent a set of stereotyped facts that, according to the experts, influence the resolution of a case. Thus, a case in CATO is a set of unary factors that are labelled as favourable to the plaintiff or to the defendant. Other important contribution of CATO was the definition of methods to generate and select arguments having the knowledge contained in the factor hierarchy into account.

Initially, CATO was deployed to work in the HYPO's domain, the trade secrets American law. However, CATO's factor-represented case-based argumentation model was used afterwards in other systems that operated in different legal domains. The system BankXX (Rissland et al., 1993), which generates arguments in the domain of American bankruptcy law by performing a best-first heuristic search in a highinterconnected network of legal knowledge, is an example. Moreover, the work done in HYPO and CATO systems also gave rise to several projects whose research objectives were centred in the development of some specific processing functionality of the legal information contained in the cases. Some examples are the systems: SPIRE (Daniels \& Rissland, 1997), which mixes CBR with retrieval information techniques to extract passages from textual legal cases that could contain relevant information of the opinion of several courts about certain legal cases; SMILE (Bruninghaus \& Ashley, 2001), which uses text classifiers to automatically decide the factors that are applicable to a specific legal case and; IBP (Bruninghaus \& Ashley, 2003), which determines the underlaying issues of a case and, on the basis of them, predicts the verdict of case-based legal processes. Recently, SMILE and IBP systems have been integrated in a new system that is able to reason directly with textual legal cases. This system analyses the cases to extract the relevant legal information, then predicts their verdicts and finally, shows such prediction with an explanation (Bruninghaus \& Ashley, 2005).

Other kind of argumentation systems that have their roots in the eighties and early nineties are the hybrid CBR-RBR (case-based and rule-based reasoning) systems. One of the first real hybrid CBRRBR system is another descendant of HYPO, the CABARET system (Rissland \& Skalak, 1991), which produces legal arguments in the domain of the taxes American law. Before CABARET's development, 
'hybrid' systems had a main reasoning mode and when it failed, the systems switched to the secondary mode of reasoning. However, CABARET has a domain-independent architecture that includes two reasoners (one case-based and other rule-based) that are managed by an agenda controller that uses heuristic rules to dynamically alternate the control over them. The system uses various knowledge sources: a database of legal cases, a database of rules and legal predicates and a set of domain-independent control rules, which determine the sequence of tasks to perform by using the information gathered by the controller. Finally, other important hybrid CBR-RBR argumentation system is GREBE (Branting, 1991), which was a pioneer system in using the justifications of legal cases to create new arguments. GREBE is a system for legal analysis that reasons with portions of precedent cases in the domain of the Texas workers compensation law. The system builds explanations for the classification of legal cases as instances of specific legal predicates by using a back-chaining technique that combines rules and portions of precedents.

\subsection{Current Applications of Argumentation in AI}

Nowadays, the argumentation research in AI is experiencing a new reactivation, mainly motivated by recent and interesting contributions developed in MAS. On one hand, the argumentation theory has been studied in MAS to manage the agent's practical reasoning. Practical reasoning is a well-known area in philosophy, but which historically has received less attention in AI than the theoretical reasoning. This type of reasoning analyses which specific action should be performed in a particular situation, instead of the theoretical reasoning objective of deciding the truthfulness of beliefs. Moreover, practical reasoning does not presuppose, as theoretical reasoning does, that the fact of reaching an objective is always adequate or profitable, but it must select the best objectives to perform and decide afterwards whether their realization is worthwhile. This fits the reality of a MAS, where each individual agent has its own point of view and its particular objectives and interests. However, the theoretical reasoning about the state of the world and the effects of the potential actions to perform is also essential. Therefore, both types of reasoning must be considered in MAS. Rahwan \& Amgoud (2006) have recently proposed an argumentation-based approach for practical reasoning. In this work, Dung's abstract argumentation framework (Dung, 1995) is instantiated to generate consistent desires and plans to achieve them. The works developed by Atkinson in her thesis and hers subsequent research are also other important contributions to the modelling of argumentation processes that allow the agents to reason about what is the best action to execute (Atkinson, 2005).

On the other hand, the argumentation techniques have been applied to manage the agents' autonomous reasoning and the interaction between them (Rahwan, 2006). In open MAS, the introduction of new information may give rise to new arguments that reinforce or weaken certain beliefs. Therefore, the argumentation techniques can be used as a way of revising the agents' beliefs in presence of incomplete or uncertain information. Capobianco et al. (2005), for example, apply argumentation to keep the consistence of the agents' mental state in changing environments by using an appropriate representation of the environment and a mechanism that integrates the new information in the beliefs update process. Argumentation has also been applied in MAS as a selection means between conflicting desires (Amgoud, 2003) and objectives (Amgoud \& Kaci, 2004), as a qualitative means of reasoning about the expected value of the realisation of certain actions (Fox \& Parsons, 1998) and as generator of plans (Hulstijn \& van der Torre, 2004), (Simari et al., 2004).

Moreover, argumentation provides MAS with a framework that assures a rational communication. The dialogue typology of Walton \& Krabbe (1995) has been adopted in MAS to classify the different types of dialogues between the agents depending on the objective of the interaction. Other concepts of the argumentation theory (i.e. dialogue games (Hamblin, 1970), (MacKenzie, 1978) and argumentation schemes (Walton, 2006)) have also been applied to structure the dialogue between agents with different points of view according to the interaction rules that have been previously agreed. Recently, a wide range of approaches that formalise interaction protocols by using different dialogue games have been published (McBurney \& Parsons, 2002). Some examples of dialogue game protocols about specific types of dialogues are: inquiry (Hulstijn, 2000), persuasion (Atkinson, 2005), negotiation (Sadri et al., 2001) 
and deliberation (McBurney et al., 2007). Finally, argumentation schemes have several characteristics that make them very useful in defining the communication between agents. In the case of Walton's argumentation schemes, the critical questions are arguments that can be presented by an opponent to criticise the claim that the scheme poses, thus providing the argument with a clear structure that reduces the computational cost of generating and evaluating arguments. In addition, Reed \& Walton (2005) propose a formal framework to specify argumentation schemes for agent's communication by using the markup language $A M L$, based on $X M L$.

Along this section has been shown how the argumentation techniques have been successfully used to reach agreements that assure the coherence of the agents' mental state and to structure their interaction in disagreement situations. Parsons et al. (1998) proposed a seminal theoretical framework that unifies argumentation-based reasoning and communication for negotiation in MAS. More recently, Rahwan et al. (2003) analyse this and other argumentation-based negotiation frameworks. A wide review of the current situation of the argumentation research in AI has also been published in the special issue on argumentation of the journal Artificial Intelligence (Bench-Capon \& Dunne, 2007). Moreover, an effort to consolidate the work done in argumentation languages and protocols, argument visualisation and editing tools and, generally, in argumentation frameworks for MAS, has been performed by the ASPIC project ${ }^{1}$. As a result, a new standard for argument interchange in MAS, the Argument Interchange Format (AIF), has been proposed to serve as a convergence point for theoretical and practical work in this area (Willmott et al., 2006). All these advances show how the study of argumentation in AI, and more concretely in MAS, is currently a research area that has a high activity and a growing interest.

Furthermore, as it was shown in the previous section, the work done in CBR argumentation systems in the eighties and nineties promoted the study of argumentation in AI. From then on, the successful application of CBR to reach agreements in case-based legal confrontations suggests the power of this methodology to manage this kind of disagreement situations. Nowadays, the argumentation research in CBR continues being very active (Rissland, 2006) and, in fact, some approaches that integrate CBR in MAS to help argumentation processes have already been proposed. However, the contributions are still scarce and many research in the area remains to be done. Our point of view is that CBR can be very useful to strategically manage argumentation in open MAS. Trying to demonstrate the foundations of this suggestion and to clarify the work that has already been done, next section reviews the uses of CBR for argumentation in MAS.

\section{Research approaches in CBR-based argumentation for MAS}

Argument management (i.e. generation, selection, evaluation etc. of the components of arguments and the management of the dialogue itself) is a key issue to deal with in argumentation-based dialogues in MAS. Our view, supported by the successful applications reported in previous sections, is that CBR is a suitable methodology to argue in two-party disagreement situations. Furthermore, if the use of CBR is extended to manage argumentation in multi-party dialogues (i.e. dialogues between a group of agents), argumentation can also be enhanced with the reasoning and learning capabilities that CBR provides. These features could promote and distinguish CBR from other approaches for argument management. Note that in what follows, our research is focused on the applications of CBR in dialogical contexts in MAS. Therefore, we assume that there are a set of agents engaged in an argumentation in a multi-agent environment. The applications of CBR to manage agents' autonomous reasoning are out of the scope of this paper.

To date, few research has cope with the use of CBR methodology to facilitate the argumentation between the agents of MAS. The current approaches are focused on managing two types of dialogues between agents: argumentation-based negotiation and collaborative deliberation. This section analyses them in an attempt to show the promising advantages of using CBR to aid argumentation in open MAS. Despite the scarcity of applications in this area, the frameworks that have been proposed still introduce important features and interesting advances to analyse.

However, the language employed by the authors to present their approaches is very varied. Therefore, before dealing with the analysis, we introduce several dimensions that aim at establishing a common

${ }^{1}$ European Union's 6th Framework ASPIC Project (IST-002307), http: / /www . argumentation . org 
terminology for our research, some of them inspired by the characteristics that Rahwan et al. (2003) used to compare several argumentation-based negotiation frameworks. These dimensions are organised and studied in three areas of analysis (i.e. context, argumentation model and discussion) and show how each framework deals with the argumentation related issues:

\section{Context:}

- Implementation domain: domain where the framework has been implemented and tested.

- Dialogue type: type of dialogue according to the classification proposed by Walton and Krabbe (1995).

\section{Argumentation Model:}

- CBR objective: what are the main purposes for applying the CBR methodology in the framework.

- Case-base contents: what kind of information is stored in the cases.

- Argument generation: method used to generate arguments in the framework.

- Argument selection: method used to select arguments in the framework.

- Argument evaluation: method used to evaluate arguments in the framework.

- Interaction protocol: method used to control the interactions between the agents of the framework.

\section{Discussion:}

- Argumentation style: theoretical framework that has inspired the argumentation style employed by the authors.

- Assumptions: main assumptions made by the authors in the design of the system that implements the framework proposed.

For clarity purposes, the analysis performed in this section is mainly centred on the CBR features that support the argumentation functionalities. The specific details about the design of each system are out of the scope of this paper and we refer the reader to the related bibliography for further information. Finally, note that not every dimension is mentioned when explaining each framework, since some of them remain unclear or unspecified by the authors.

\subsection{The PERSUADER system}

\section{Context}

In her thesis, Katia Sycara developed the PERSUADER system, which acts as a mediator in the implementation domain of labour management disputes between a company and its trade union (Sycara, 1987, 1989, 1990). This was a seminal framework that integrated for the first time concepts of argumentation theory and CBR to create a negotiation model in a MAS. PERSUADER uses a mediator agent that manages the negotiations between two agents representing the company and the trade union. The mediator dialogues with the parts trying to reach an agreement, which is a contract that is accepted by both agents. A contract consists of a set of attributes (e.g. salaries, pensions and holidays) whose value must be decided. Opposite to many systems of its time, PERSUADER studied the argumentation in a non-cooperative domain, where each agent has its own objectives and tries to derive its maximum own benefit from the negotiation. The main objective of the mediator and hence, the objective of the dialogue in this framework, is to negotiate with both agents and persuade them to collaborate.

\section{Argumentation Model}

The agents' negotiation model consists of: (a) an iterative process that implies the identification of potential interactions (either by communicating with the other agents or by reasoning about the current state of the negotiation and the agents' intentions) and (b) the modification of the own intentions to avoid the interactions that could hinder the agreement process. Thus, the model favours the cooperation. In 
addition, the mediator agent is able: (a) to represent and keep models of the agents' beliefs and preferences, (b) to reason with these beliefs and (c) to modify them to influence their behaviour. Therefore, in order to perform a persuasive negotiation, the mediator agent keeps a model of the company and trade union agents and by reasoning over it, tries to find all possible ways of affecting the behaviour of these agents.

In PERSUADER, one of the CBR objectives is to infer the model of beliefs and preferences of an unknown agent. In this way, the mediator retrieves the information about past negotiations with similar agents that was stored in precedent cases and adapts it to fit to the current context. Moreover, during the negotiation, the mediator agent can update the agents' models by observing their reactions to the arguments that it offers to them. To manage the negotiation process, the mediator agent has available two types of knowledge:

- Domain knowledge stored in the case-base: the contents of the case-base consist of negotiation concepts represented by attributes that are hierarchically organised in networks (i.e. negotiation issues, concrete negotiators, negotiators' objectives, negotiation context and final agreement).

- Reasoning knowledge: is the knowledge needed to evaluate the fairness of a contract or to improve it. This knowledge is represented in terms of multi-attribute utilities that are associated with the objectives of each agent and express its preferences and criteria for selecting among proposals.

The argument generation is performed by integrating several techniques: the search in an objective graph, the use of the multi-attribute utilities and the use of the case-base of precedent negotiations. In PERSUADER, persuading an agent to change its assessment about the proposal of certain contract entails producing an argument that increases the benefits that this agent receives with that contract. The received benefits can be inferred as a linear combination of the utilities that will be received for the value of each attribute that composes the contract. This also determines whether the objectives of the agent have been fulfilled or not. Therefore, the global benefit that will receive an agent can be increased (and hence, the agent can be persuaded) by following two types of persuasion strategies:

1. Strategies to change the importance of a concrete objective:

- To indicate a change (increment or decrement) in the contribution of the objective to fulfil an objective of highest level.

- To indicate a change in the viability or efficiency of the objective.

2. Strategies to change the utility value of a concrete objective:

- To retrieve from the case-base a counterexample that shows an opposite behaviour of the agent in a similar past negotiation.

- To retrieve from the case-base examples of similar agents that accepted the proposed value of the objective in similar past negotiations.

Therefore, another CBR objective in PERSUADER is to retrieve past cases that act as arguments for persuading an agent to accept a specific contract. In case that one part tried to prematurely withdraw from the negotiation without having reached an agreement, in addition to these appealing arguments, the system is able to generate threats.

The negotiation process in PERSUADER starts with the introduction of the set of objectives of the company and the trade union that conflicts and the factors that define the negotiation context. Initially, the mediator agent generates a contract proposal and shows it to the agents. If the proposal is accepted, the negotiation ends. Otherwise, the mediator chooses either to generate another contract proposal (if none of the parts accept it) or to start a persuasive argumentation trying to persuade the agent that does not accept the proposal (if it is accepted by one of the agents). The output of the negotiation is the agreement of a contract or an indication that the negotiation failed after certain number of proposals.

\section{Discussion}

The PERSUADER argumentation style is based on the persuasion psychology of Karlins \& Abelson (1970) and, as it is pointed out before, there are two types of possible arguments: appealings or threats. 
The main contribution of the system was the creation of the first model of persuasive argumentation between several, by default, non-cooperative agents with different interests that integrates elements of CBR, utility theory and argumentation theory. However, this was a foundational work where the agent's model was somehow specified by abstractions. Although the author justified such design decision by saying that it reduces the overload of building and maintaining real agent's models, the multi-agent condition of the system could be debatable. Anyway, the system is completely designed for two party mediated argumentation, which is inapplicable to open MAS, where the number of participants in the argumentation dialogue can change and the presence of a mediator cannot be assumed by default. Moreover, PERSUADER is highly domain-dependent and user-oriented, with reasoning algorithms based on the knowledge introduced on the system, argument selection completely determined by a preestablished hierarchy of argument types and user-based evaluation of arguments.

\subsection{CBR for Argumentation with Multiple Points of View}

\section{Context}

Nikos Karacapilidis et al. developed a model that integrates CBR and argumentation for supporting decision making in discussion processes. This model was implemented in the Argument Builder Tool $(A B T)$ of the multi-agent framework for collaborative deliberation HERMES (Karacapilidis \& Papadias, 2001), (Karacapilidis et al., 1997). This is an Argumentation-based Decision Support System (ADSS) that helps a group of users (human agents) to build sound arguments to defend their positions in favour or against other alternative positions in a discussion. HERMES maps the argument process into a discussion graph with tree structure and shows graphically the possible discourse acts that the agents could instance. The system uses CBR to make the appropriate queries to the (internal or external) databases that store information that support the positions of the agents that participate in the argument and, thus, to generate discourse acts that successfully show their interests and intentions. However, as it is only a support system, afterwards the agents are free to adopt or not the ABT's proposals.

\section{Argumentation Model}

In this framework is the system itself who manages the interaction between the agents, being the CBR engine a reasoning component integrated in it. Therefore, the case-base is common for all agents and belongs to the system. The cases are flexible entities that store a set of argumentation elements that can be interpreted depending on the state of the discourse and each agent's point of view. The argumentation elements that HERMES considers are the following:

- Issues: decisions to take or objectives to fulfil. They consist of a set of alternatives.

- Alternatives: potential choices.

- Positions: predicates that either advocate for the choice of a concrete alternative or deviate the agents' interest for it. They can also refer to other positions and give additional information about them.

- Constraints: which represent preference relations over arguments $(<$ position, preference relation, position $>$ ), where the preference relation can be more (or less) important than or of equal importance to certain alternative.

The arguments in HERMES are either tuples $<$ position, link, position $>$ or $<$ position, link, alternative $>$, where a positive link denotes an argument favouring an alternative and a negative link denotes a counterargument to it. The system evaluates them by using constraints that the users of the system introduce, checking previously the consistence of the new constraints in relation with the constraints that have been introduced before. This checking gives rise to new argumentation processes to solve the possible conflicts between constraints. The constraints establish a partial ordering over the positions and, in this way, the position with a highest weight (highest preference) becomes the winning argument.

The argumentation process in HERMES is performed by means of several discourse acts with different functions and roles. There are two types of discourse acts: (1) agents' acts, which represent the user's actions and correspond to the functions that the user interface of HERMES supports (e.g. opening of new issues, alternatives submission, etc.) and, (2) system's internal acts, which are consequence of the 
agents' acts and represent functions for discourse consistency checking, discussion update and solution recommendation. The latter functionality is performed by using CBR.

Therefore, the main objective of the CBR methodology in the system is to examine the current discussion and to suggest the participants the best discourse acts to fire, according with their points of view and preferences. Thus, the contents of the HERMES case-base represent past argumentation processes. The cases consist of the following elements:

- Situation: relevant part of the discussion at the time when the case was stored in the case-base. It characterises the concrete problem that the case represents and consists of the elements:

- Target: element to be argued about by the agent (issue, alternative or position).

- Discussion: discussion that the case has been extracted from (represented by a link to the relevant part of the discussion tree that the case refers to).

- Solution: alternative or position that the system proposes to argue about the target.

- Evaluation: suitability of the case to the agent's agenda.

The case-based argumentation process in HERMES consists of the following phases:

1. Intention submission: first, agents submit their intentions by declaring the arguments that they want to include in the argumentation and by mapping their point of view about the current discussion. Therefore, the arguments are manually generated by instantiating some of the pre-established discourse acts that the system has.

2. Cases retrieval and selection: then, HERMES retrieves those cases for which the target coincides with the agents' current argumentation objective. Afterwards, the system performs a case selection based on the agents' point of view and the current state of the argumentation.

3. Cases adaption: this is a semi-automatic process, being the user who selects the cases that need adaption among the set of similar cases that the system has proposed.

4. Argument assertion: finally, by using the retrieved and adapted cases, the agents provide warrants to their assertions. Argument assertion involves firing the appropriate discourse acts to propagate the information in the discussion graph and to retain the new case.

\section{Discussion}

The argumentation style of HERMES is inspired by the ZENO's model of argumentation (Gordon \& Karacapilidis, 1997), which is based on the IBIS informal-logic argumentation framework (Rittel \& Webber, 1973). Therefore, HERMES interprets arguments on the basis of their intentional context by using an informal logic. The authors view the system as a MAS because it can be used by several human agents that interact between them and with the system by means of the HERMES user interfaces. An important contribution of HERMES was the proposition of this new case-based argumentation support functionality, which, in view of the discussion current state, produces the best arguments to support the assertions of the participants taking their points of view and preferences into account. Moreover, the user interface allows to follow easily the course of the argumentation. However, the framework does not define a specific interaction protocol between the agents. In addition, the arguments evaluation depend on constraints that determine a preference relation among them, also introduced by the users. Therefore, the good-end of the argumentation process entirely depends on the honesty, experience and disposition to collaborate of the users, which cannot be assured in open environments. Human intervention is compulsory almost at any time. These assumptions pose heavy difficulties to adapt this framework to work in open MAS with a changing number of heterogeneous software agents participating in the dialogue.

\subsection{Case-based Negotiation Model for Reflective Agents}

\section{Context}

Leen-Kiat Soh and Costas Tsatsoulis designed a case-based negotiation model for reflective agents (i.e. agents aware of their temporal and situational context). This model uses CBR to plan/re-plan the 
negotiation strategy that allows the most effective negotiation on the basis of past negotiations (Soh \& Tsatsoulis, 2001a, 2001b, 2005). In this framework, a set of situated agents that control certain sensors try to track several mobile targets. The aim of the agents is to coordinate their activities and collaborate to track the path to as many targets as possible. The agents' sensors have limited power and coverage and each agent only controls a subset of sensors. Although the cooperativeness is assumed, each agent has individual tasks to fulfil. Therefore, when an agent has not enough coverage or power capabilities to track a target, it needs to negotiate and persuade other agents and achieve that they leave their tasks and help it to track the target. Hence, the framework was implemented to solve a typical problem of limited resources allocation.

\section{Argumentation Model}

The agents of this model are autonomous entities that own two separated and private case-bases. Each agent has a $C B R$ manager that performs 3 functions: (1) retrieves past negotiation cases that are similar to the current negotiation, (2) uses them to determine new negotiation strategies and (3) maintains the casebase. The CBR manager allows agents to learn to negotiate more effectively by using the knowledge of past negotiations. The cases contents store descriptions that characterise the agents' context in a previous negotiation. Concretely, a case is composed by the following elements:

- Description of the part of the world relevant to the case: information about the sensors and the target.

- Agent's profile: sensor power, activated sensor sector, state of the communication channels, task list, etc.

- Neighbours' profile: agent's knowledge about its neighbours.

- Negotiation outcome.

An agent can play two possible roles: as initiator or as responder of the negotiation. As initiator, the agent tries to negotiate with its neighbours to reach its targets. As responder, the agent receives a negotiation proposal and answers it. Therefore, the information gathered by the agent when playing each role is stored in the corresponding case-base. When an agent determines that it cannot reach a target by its own, it tries to collaborate with other agents initiating an argumentation dialogue. In this framework, the arguments are pieces of information that an agent sends to its neighbours to persuade them to share certain resource with it. Persuasion implies to surpass the negotiation threshold of an agent and convince it to share some of its resources.

In order to achieve it, the initiator agent retrieves cases that represent negotiations that are similar to the current one from its initiator case-base. The cases are retrieved by comparing their case descriptors with the characteristics of the new negotiation. The negotiation strategy that will follow the agent, which is deduced from the negotiation parameters of the cases, specifies which type of information should be sent to convince quickly a particular responder agent. Initially, the initiator agent retrieves several strategies and afterwards, it uses Multivalued Utility Theory. By using this theory agents are able to relate constraint satisfaction criteria of the negotiation at hand with the potential negotiation strategies and to select the one that optimises such criteria while minimising the risky behaviour.

In this model the cases are situated, thus, the CBR manager must adapt the negotiation parameters to fit to the current negotiation. To that purpose, it uses certain domain-dependent adaption rules that the framework pre-establishes and, once the adaption is performed, the negotiation starts. The arguments to send to other agents are ranked and selected on the basis of certain selection rules also pre-established in the framework. Following them, the initiator agent selects which piece of information (arguments about the world, the target or the agent itself) can be most effective to persuade a specific agent in the current context. In each negotiation step, the initiator agent sends an argument to the responder agent. In its turn, the responder agent evaluates the evidence of each argument by using the information of its responder case-base and a relaxed constraint satisfaction approach. The process continues until a maximum number of steps is reached or an argument surpasses the negotiation threshold of the responder agent and it is accepted. The interaction protocol is defined by means of a series of states over which the negotiation takes place. At any time, the negotiation parameters that the CBR manager adapts have a high influence in the sequence of states that the negotiation follows. 


\section{Discussion}

The framework of Soh and Tsatsoulis does not interpret persuasion as most legal argumentation systems do (Jakobovits \& Vermeir, 1999). These perform a detailed study about the dialectical context of legal arguments and their condition of proposals, defences, positions or attacks. However, the argumentation style of this framework views persuasion as a negotiation protocol of information interchange between two agents that try to reach an agreement by using an argumentation process. To describe the logical framework, the multicontext BDI framework of (Parsons et al., 1998) was extended. In addition, temporal characteristics were included by using a temporal logic that defines relations over time intervals. Other important contribution of this framework was the introduction of learning capabilities for the agents by using the CBR methodology. Moreover, the negotiation strategy is inferred dynamically from the information of the case-base and concurrent negotiations are allowed.

However, the model assumes certain characteristics that can pose several drawbacks to its application to open MAS. On one hand, the neighbours and their controlled sensors must be known in advance. On the other hand, despite concurrency is admitted, the agents can only negotiate about one issue at the same time. Finally, the framework has strong assumptions about the honesty, cooperativeness and rationality of the agents that do not fit the reality of open MAS.

\subsection{Argument-based selection Model (ProCLAIM)}

\section{Context}

Pancho Tolchinsky et al. extended the architecture of the decision support MAS for the organ donation process CARREL+ (Vazquez-Salceda et al., 2003) with ProCLAIM, a new selection model based on argumentation (Tolchinsky et al., 2006a, 2006b, 2006c). In CARREL+, a donor agent (DA) and a set of recipient agents $(R A s)$ argue about the viability of the organ transplant to some recipient. If an agreement is not reached, the organ is discarded. ProCLAIM includes a mediator agent $(M A)$ that controls the collaborative deliberation dialogue and uses a CBR engine to evaluate the arguments about organ viability that the agents submit. The final decision must fulfil several guidelines that, in ProCLAIM case, are the human organs acceptability criteria that CARREL stores in the Acceptability Criteria Knowledge Base $(A C K B)$.

\section{Argumentation Model}

The mediator agent uses a case-base to store all relevant information about past donation processes. The cases contents consist of a description of the transplant medical information and an argument graph that shows the arguments that were submitted by the agents that participated in the donation process. Different cases can share the same argument graph. In addition, an argument graph has an evidential support $\langle F, K\rangle$, where $\mathrm{F}$ stands for the certainty on the correctness of the final decision that was made and $\mathrm{K}$ for the number of cases that share the graph. This certainty corresponds to the transplant phase when the decision was made; phase 1 includes potential arguments that were submitted before the organ extraction, phase 2 includes conclusive arguments that were submitted after the organ extraction and phase 3 includes conclusive arguments about the final result after the organ transplant.

The MA tasks consist of (1) directing the possible dialectical movements of each agent, (2) ensuring that the submitted arguments observe the guidelines and (3) using CBR to assign intensities to the arguments, offer new relevant arguments and take a final decision about the winning arguments. ProCLAIM arguments are instantiations of the argument schemes and critical questions of the CARREL+ knowledge resource Argument Scheme Repository (ASR). This repository completely characterises the space of possible arguments that the agents can submit. Moreover, agents use a 1st order logic programming language to generate arguments (Modgil et al., 2005). When a new organ is offered, the DA submits to the MA its arguments about the organ viability. Afterwards, the RAs counterargue and the MA must take a final decision. To that moment, the arguments are of phase 1. Once the organ is extracted, a RA can change its mind and consider it as non-viable. Therefore, the RA must submit more phase 2 arguments to support its position. Finally, if the organ is transplanted and complications arise, the RA submits more 
phase 3 arguments to explain the failure or the actions performed to solve them and achieve a successful transplant.

When the DA and the RAs have submitted their arguments for the organ transplant, the MA evaluates them. If there are conflicts, the MA compares the resulting argument graph with those stored in its casebase to retrieve similar graphs and decide the winning arguments. Once the similar argument graphs are obtained, the MA checks the descriptors of the cases that the graphs have associated and rules out the ones that contain arguments that do not observe the guidelines. In addition, the graphs whose evidential support falls below certain threshold are also rejected. In some exceptional cases, the CBR engine could find valid arguments that were turned down by the guidelines. Even arguments that were rejected by both the CBR engine and the guidelines can be accepted because the agent who submitted them has either a high reputation, a specific role that increases the confidence on its opinion or a certificate that supports its arguments. This knowledge is encoded in the Argument Source Manager (ASM) resource and can be acceded by the MA to readjust the strengths of the arguments. The potential conflicts between the evaluation of arguments proposed by the different CARREL+ knowledge resources are solved with a pre-established resource preference relation. Finally, the resulting set of argument graphs is put together in a solution graph that represents the CBR engine decision concerning the viability of the transplant to a specific recipient agent. If this argument graph was already included in the mediator's case-base, the description of the new case is associated with it and, hence, its evidential support increases. Otherwise, the new graph and its associated case are added to the case-base.

\section{Discussion}

ProCLAIM argumentation style is based on Dung's abstract argumentation framework. An interesting contribution of this model is that it can increase the organ acceptance rate by allowing the donor and the recipients to argue about their decisions. In addition, it also allows exceptions over the human organ transplant acceptability guidelines. The ProCLAIM model has also been recently applied to wastewater management (Aulinas, 2007) (also a collaborative decision making domain with critical features) and to deliberate over action proposals (Tolchinsky et al., 2007). However, in all applications the argumentation process depends on the contents of the knowledge resources that the MA accedes. Therefore, an intensive effort to acquire and maintain such knowledge must be performed. Moreover, as pointed out before, the space of possible arguments to submit is completely characterised by the ASR. This implies that the agents have limited expressiveness, although the authors state that this decision has been taken for security reasons in the critical domains where the model operates. As Sycara's PERSUADER system, this is also a framework that was designed for performing a mediated argumentation, but opposite to PERSUADER, the cooperative nature of the agents is assumed by default. Therefore, this heavy assumption hinders again the adaption of this system to open MAS.

\subsection{Argumentation-based Multi-Agent Learning (AMAL)}

\section{Context}

Santiago Ontañón and Enric Plaza developed the Argumentation Based Multi-Agent Learning (AMAL) framework (Ontañón \& Plaza, 2006, 2007). The agents of this framework are autonomous entities able to independently solve classification problems and to learn by experience, storing the knowledge acquired during the solving process in their private case-bases. The set of possible classification classes is predefined in the framework. The aim of the interaction between the agents is to increase the solution quality by aggregating the knowledge of a group of expert agents. Therefore, they engage in a collaborative deliberation dialogue.

\section{Argumentation Model}

When an agent receives a new problem to solve, it firstly uses the CBR methodology to retrieve the most similar cases from its case-base and provide an initial solution to the problem. In this way, the problem is classified into the class that the most similar cases belong. In this framework, the cases contents consist of a set of attributes that describe a problem and the solution class that classifies it $(C=<P, S>)$. Moreover, 
the AMAL agents are able to provide a justified prediction $(J=<A, P, S, D>)$ that explains the reason why a certain solution has been proposed to solve a specific problem. An agent $A$ generates the justified prediction $J$ to assert its belief that $S$ is the correct solution for the problem $P$ and $D$ is the evidence support for such statement. This evidence is a symbolic description that contains the relevant information (common attributes) shared by the problem and the retrieved cases whose class is $S$. Therefore, $D$ stands for the claim that all or most of the cases that are similar to $P$ in the agent's case-base belong to the class $S$. The justified predictions can be generated by using CBR in combination with any other learning method, as decision trees or Lazy Induction of Descriptions, LID (Armengol \& Plaza, 2001), the latter being the method used in AMAL.

Both cases and justified predictions are information pieces that AMAL agents use to generate three types of arguments:

- Justified Predictions, as previously explained.

- Counterarguments, which show the evidence that other agent has for classifying the problem as belonging to a different class.

- Counterexamples, which are cases sent by other agent to contradict an argument by showing an example case that classifies the problem into a different class.

By means of the arguments, agents get involved in a global solving process that increases the quality of the initial solutions proposed by each agent by reaching an agreement about the correct solution for the problem. To achieve this, a preference relation to evaluate the arguments that bring into conflict is needed. In AMAL, this preference relation is based on a global confidence measure that is computed for each justified prediction. In the face of several conflicting arguments, the winning argument is that with higher global confidence (the one that classifies the problem into the class that has been predicted by the major number of agents).

The deliberation process in AMAL takes place across an interaction protocol that defines a series of rounds. In each one a token passing mechanism is used to specify which agent is authorised to interact with the others. During its round, an agent can either assert an argument or rebut it with a counterargument (or counterexample). Agents can also assert arguments when they accept an incoming argument and change their prediction. The AMAL protocol allows agents to include the counterexamples that receive in their case-bases and, hence, to increase their knowledge. The interaction ends when an agreement is reached or when a maximum number of rounds is surpassed. In the latter case, the final solution for the problem is decided by using a weighted voting mechanism.

\section{Discussion}

The AMAL framework is a newly contribution to the study of argumentation-based learning models for MAS whose agents have individual learning capabilities. This model also differs from many other argumentation frameworks on its dynamic computation of the relation preference between arguments. In addition, the argumentation style is completely case-based. However, the framework assumes honesty, cooperativeness and rationality in the agents' interactions, which cannot be assured in open MAS. All agents must have at least some knowledge about the problem to solve. Therefore, this framework is not conceived for open distributed environments, where the heterogeneity of agents makes non-assumable that all participants in a dialogue have a minimum knowledge about the problem at hand. Obviously, in order to take the maximum profit from this approach of learning from communication, the knowledge must be conveniently distributed over the agents' case-base. If all agents become experts, collaboration and learning from others would be a nonsense.

\subsection{Conclusions}

The examples about systems that successfully apply CBR to manage argumentation in MAS demonstrate the suitability of this reasoning methodology to provide agents with the ability to argue. However, a formal and context-independent framework that defines a strategic argumentation theory for agents with learning capabilities does not already exist. The main properties of the frameworks that this paper has reviewed 


\begin{tabular}{|c|c|c|c|c|c|}
\hline $\begin{array}{|ll|}\text { Dimensions } & \text { Framework } \\
\end{array}$ & PERSUADER & HERMES & SOH's & PRoCLAIM & AMAL \\
\hline \begin{tabular}{l|l} 
Implementation domain \\
\end{tabular} & $\begin{array}{l}\text { Labour conflicts } \\
\text { mediation }\end{array}$ & $\begin{array}{l}\text { Decision support } \\
\text { (e.g. Deciding } \\
\text { treatments for } \\
\text { patients and } \\
\text { Planning cyclepaths) }\end{array}$ & $\begin{array}{l}\text { Dynamic resource assignment in a } \\
\text { sensorised environment with } \\
\text { mobile targets }\end{array}$ & $\begin{array}{l}\text { Decision support (e.g. Organ } \\
\text { transplant and Wastewater } \\
\text { management) }\end{array}$ & Classification problems \\
\hline Dialogue type & $\begin{array}{l}\text { Negotiation \& } \\
\text { Persuasion }\end{array}$ & Deliberation & Negotiation & Deliberation & Deliberation \\
\hline CBR objective & $\begin{array}{l}\text { Provide information } \\
\text { for building agent's } \\
\text { models. Generate } \\
\text { precedent-based } \\
\text { arguments }\end{array}$ & $\begin{array}{l}\text { Provide argument } \\
\text { warrants. Assist in } \\
\text { argument generation }\end{array}$ & Select negotiation strategies & $\begin{array}{l}\text { Argument evaluation. Offer other } \\
\text { relevant arguments }\end{array}$ & $\begin{array}{l}\text { Individually solve the } \\
\text { problem. Select the global } \\
\text { solution. Learn by } \\
\text { experience }\end{array}$ \\
\hline Case-base contents & $\begin{array}{l}\text { Concepts of previous } \\
\text { negotiations } \\
\text { hierarchically } \\
\text { organised in networks }\end{array}$ & $\begin{array}{l}\text { Set of attributes } \\
\text { representing past } \\
\text { argumentation } \\
\text { processes }\end{array}$ & $\begin{array}{l}\text { Descriptions of the agents' context } \\
\text { in a previous negotiation }\end{array}$ & $\begin{array}{l}\text { Medical information of past } \\
\text { transplants and argument graph of } \\
\text { their donation processes }\end{array}$ & $\begin{array}{l}\text { Description of previous } \\
\text { problems and their solution } \\
\text { class }\end{array}$ \\
\hline Argument generation & $\begin{array}{l}\text { Case-based, objective } \\
\text { graphs search and } \\
\text { multiattribute utilities }\end{array}$ & Manual generation & Multivalued attribute utility theory & $\begin{array}{l}\text { Instantiation of the argument } \\
\text { schemes and critical questions of } \\
\text { Walton (1996). 1st order logic } \\
\text { programming language (Modgil et } \\
\text { al., 2005) }\end{array}$ & $\begin{array}{l}\text { Lazy Induction of } \\
\text { Descriptions (Armengol \& } \\
\text { Plaza, 2001) }\end{array}$ \\
\hline Argument selection & $\begin{array}{l}\text { Pre-established } \\
\text { conviction power } \\
\text { hierarchy }\end{array}$ & $\begin{array}{l}\text { Helped by the ABT } \\
\text { proposals }\end{array}$ & Domain-dependent selection rules & $\begin{array}{l}\text { Preference relation among } \\
\text { knowledge resources }\end{array}$ & $\begin{array}{l}\text { Selection implicit in CBR } \\
\text { cycle }\end{array}$ \\
\hline Argument evaluation & User-based evaluation & $\begin{array}{l}\text { User-based } \\
\text { evaluation }\end{array}$ & $\begin{array}{l}\text { Constraint relaxed satisfaction. } \\
\text { Context-dependent rules }\end{array}$ & $\begin{array}{l}\text { Guidelines-based and } \\
\text { precedent-based evaluation } \\
\text { performed by the MA }\end{array}$ & $\begin{array}{l}\text { Preference relation based } \\
\text { on a case-based dynamic } \\
\text { confidence measure }\end{array}$ \\
\hline Interaction protocol & $\begin{array}{l}\text { Non-specified. } \\
\text { Defined by the } \\
\text { mediator's decisions }\end{array}$ & $\begin{array}{l}\text { Non-specified. } \\
\text { Defined by the } \\
\text { discourse acts }\end{array}$ & $\begin{array}{l}\text { Defined by the state diagram. } \\
\text { Influenced by the CBR manager } \\
\text { decisions }\end{array}$ & $\begin{array}{l}\text { Guided by the MA. Dialogical } \\
\text { movements determined by the ASR } \\
\text { argument schemes instantiation }\end{array}$ & $\begin{array}{l}\text { Argumentation-based } \\
\text { Multi-Agent Learning } \\
\text { Protocol }\end{array}$ \\
\hline Argumentation style & $\begin{array}{l}\text { Karlins persuasion } \\
\text { psychology (Karlins \& } \\
\text { Abelson, 1970) }\end{array}$ & $\begin{array}{l}\text { Zeno's informal } \\
\text { logic (Gordon \& } \\
\text { Karacapilidis, 1997) }\end{array}$ & $\begin{array}{l}\text { Persuasion. Parson's et al. logic } \\
\text { framework (Parsons et al., 1998) }\end{array}$ & $\begin{array}{l}\text { Dung's argumentation framework } \\
\text { (Dung, 1995) }\end{array}$ & $\begin{array}{l}\text { Case-based multi-agent } \\
\text { learning }\end{array}$ \\
\hline Assumptions & $\begin{array}{l}\text { Human agents. Unique } \\
\text { mediator's case-base }\end{array}$ & $\begin{array}{l}\text { Unique system } \\
\text { case-base. Human } \\
\text { agents. CBR is a } \\
\text { system component }\end{array}$ & $\begin{array}{l}\text { Shared ontology, Agents are } \\
\text { Homogeneous, cooperative, } \\
\text { autonomous, reflective, honest and } \\
\text { rational. } 2 \text { private case-bases per } \\
\text { agent }\end{array}$ & $\begin{array}{l}\text { Agents are collaborative. Control } \\
\text { centralised by the MA. Unique MA's } \\
\text { case-base }\end{array}$ & $\begin{array}{l}\text { Shared ontology, } \\
\text { cooperative and } \\
\text { autonomous agents, shared } \\
\text { objective. One private } \\
\text { case-base per agent }\end{array}$ \\
\hline
\end{tabular}

Table 1 Main features of the analysed CBR-based argumentation frameworks.

are summarised in the Table 1 (referring the dimensions previously presented and analysed in section 3 ). The diversity of domains and purposes in applying both argumentation and the CBR methodology on each framework makes difficult to perform a formal comparison that expresses which is better at solving a particular type of problems. Therefore, the table aims at summarising and clarifying how each framework deals with the argumentation related issues. Nevertheless, some similarities and differences between them can still be identified.

As pointed out before, the implementation domain differs almost on each framework, being HERMES and ProCLAIM the ones that somehow share a common purpose: to provide decision support for a group decision-making. In addition, among other applications, both have been implemented and tested in the medical domain (Karacapilidis \& Papadias (2001), Tolchinsky et al. (2006c)). In this dimension, the main difference between them is that HERMES helps agents to select the best argument to instantiate in a particular context and hence, to win the discussion, while in ProCLAIM the system assists the mediator agent (and not the donor agents) to decide which agent has posed the best argument and should be the winner of the discussion. Therefore, although working in a similar domain, these systems are aimed at solving different subproblems inside the more general problem of supporting group decision-making.

Similarly, although HERMES, ProCLAIM and also the AMAL framework share the same dialogue type (i.e. deliberation), the final objective of the interaction between the agents of these systems is quite different: HERMES is mainly centred on the argument diagramming and its graphical representation, helping agents to follow the discussion and supporting them with tools to pose better arguments; ProCLAIM deals with the internal deliberation of the mediator agent, supporting only this agent to make the best decision among the set of potential winners and finally; in the AMAL framework all agents have the common objective of deciding the best classification tag for a specific object and act as a group of experts that cooperate by aggregating their knowledge in the deliberation process. In the same way, PERSUADER and Soh's frameworks also share the dialogue type (i.e. negotiation), but from a different perspective. Thus, while in PERSUADER the mediator agent completely centralises the negotiation process and the company and the trade union do not keep a direct interaction, in Soh's framework all agents are autonomous and able to play an initiator role that starts and manages a direct dialogue with other agents. 
With respect to the CBR objective, in all frameworks the CBR methodology has been mostly used to generate, select or evaluate arguments on the face of previous similar experiences. Consequently, as in any CBR system, the contents of the case-base in each framework consist of a set of elements that describe these previous experiences. However, the power of case-based learning can be better well-spent and could be used, for example, to perform a strategic argumentation. PERSUADER and Soh's frameworks have proposed the first preliminary attempts to develop case-based argumentation strategies by learning agent's profiles and hence, easily persuading specific types of agents. In these frameworks, the contents of the agent's case-base store some features about other agents that were observed during previous negotiations. These features are used afterwards to enhance similar negotiations by having agents' beliefs and preferences into account (e.g. PERSUADER) or by sending the most useful information to persuade a specific agent (e.g. SOH's framework). However, in both frameworks the application domain has a decisive influence on the argumentation strategy. Thus, in PERSUADER arguments are presented following a domain dependent argument hierarchy while in Soh's framework cases are situated and need domain-specific adaption rules to devise strategies that are applicable to the current negotiation context from them. As will be shown in the next section, argumentation strategies have to be further elaborated and a lot of future work remains to be done in this area.

Moreover, the application domain of each concrete framework and the contents of the case-base have a decisive influence in the entire argument management process (i.e. case-based generation, selection and evaluation of arguments). Only the AMAL framework performs a completely case-based argument management. Note that the agents of this framework use their case-base at every step of the argument management process (although they generate arguments by using the LID technique (Armengol \& Plaza, 2001), this method makes use of the information stored in the cases). The other frameworks include different techniques to manage the generation, selection or evaluation of arguments (or the user manually performs them). Furthermore, in PERSUADER, HERMES and ProCLAIM, all responsibility about the management of the argumentation process falls on one agent or on the system itself, while the rest of participants in the dialogue are human entities that are only in charge of posing arguments in favour of their objectives. It is not demonstrated that the methods and argumentation theories that are valid for a single agent were also valid if the system was entirely automated by creating really autonomous software that act on humans behalf. On most cases, the argumentation that is conducted by a mediator totally depends on its capabilities and features. Therefore, multi-agent argumentation and moderated argumentation could need from different argumentation theories and methods with every likelihood. The differences between both types of argumentation can be even greater when working with open MAS.

In the argumentation style dimension, all frameworks differ. On one hand, although PERSUADER and Soh's frameworks are based on the theory of persuasion, the former follows a formal psychological theory proposed by Karlins and Abelson (1970), which studies the different types of arguments that are thought to be persuasive in negotiation processes between humans, while the latter views persuasion as a negotiation protocol to coordinate the interaction between two agents that want to reach an agreement in a resource allocation problem. This protocol is based on the formal argumentation framework for negotiation and reasoning proposed by Parsons et al. (1998). On the other hand, the frameworks intended for deliberation also show important differences in the foundations of their argumentation style: HERMES is based on Zeno's informal logic, which proposes an argument-based labelling function to evaluate the quality of the potential positions proposed as solutions in a group decision-making problem; ProCLAIM uses Dung's acceptability criteria (Dung, 1995) to determine the winning arguments among the set of all proposed ones; and the AMAL framework follows an informal argumentation theory where cases are used to create the different argument types of the framework and also to evaluate the the preferred ones.

Finally, regarding the main assumptions made by the frameworks, PERSUADER and HERMES mostly rely on the existence of human agents that interact with the system. In the case of PERSUADER, this fact is probably due to the time when the framework was developed, when multi-agent systems were still in their early beginnings. In HERMES' case, the focus of the system on developing a web interface to support group decision-making and not on replacing human judgement motivates this strong assumption on the existence of human users. In addition, most frameworks assume collaborative or cooperative agents and 
do not tackle with reputation and trust issues. In fact, none of the reviewed frameworks takes into account the possibility to allow malicious and interested agents to come into the system. In this case, the honesty and cooperative predisposition of these agents cannot be assured. The agents of Sycara's PERSUADER system are not cooperative by default, but nonesty and rationality in their actions are still assumed. In the ProCLAIM model, the MA can accede to the ASM to evaluate the arguments submitted by an agent in view of its reputation. However, the concept of reputation here seems to be a pre-defined value for each agent that stands for its expertise in a specific domain. Again, malicious behaviours are not prevented. Therefore, all these frameworks are not intended for operating in open environments with heterogeneous and possibly unreliable agents.

\section{Open Research Issues}

In previous sections different CBR systems for argumentation have been analysed, trying to identify their important contributions and the research challenges that are dealt with. Based on this analysis, we have specified several broad areas that the research community has to further investigate. This section poses open issues in these areas that must be tackled in order to promote the work on argumentation in open MAS with CBR capabilities. Theses open issues offer a wide range of reseach possibilities to produce interesting advances in CBR, argumentation and open MAS. However, they are not intended to be a comprehensive list of all possible applications of CBR in the wide field of multi-agent argumentation systems, but a set of purposes for promoting new research in the area.

\subsection{Case-based Argument Representation}

A fundamental decision to make in the design of a CBR system that will determine its final operation is the case structure and contents. Bylander and Chandrasekaran stated the interaction problem (Bylander \& Chandrasekaran, 1988) by which:

'...representing knowledge for the purpose of solving some problem is strongly affected by the nature of the problem and the inference strategy to be applied to the problem...'

In the argumentation domain, how to reason about arguments, how to interpret them and how to represent their relations are key issues. A CBR system whose purpose is to perform argumentation tasks in MAS must facilitate the reasoning in this domain. Therefore, the underlaying semantics of argumentation dialogues must be taken into account when deciding the structure and the representation language of the cases.

If the arguments that have been submitted in a dialogue are only stored as data in cases of the type attribute-value, or similarly, if the cases are simple structures that store information that will be used later to generate arguments, the semantic knowledge acquired during the argumentation is lost. This knowledge could be crucial to develop argumentation strategies able to persuade, for instance, agents with specific profiles or to enhance the interaction protocol (see section 4.2). As pointed out in section 3.6, PERSUADER and Soh's frameworks made the first steps to devise argumentation strategies from the information stored in the cases. In both frameworks, cases store information about the context of previous argumentation processes (e.g. participants' objective, environmental data, negotiation issues, final agreement, etc.). However, the structure and representation language of the cases do not allow to define semantic relations between such concepts.

In addition, if general argumentation knowledge is stored in the cases together with the information about previous argumentation processes (i.e. arguments or information pieces to generate them), this knowledge could ease its later interpretation. Our suggestion is to use Knowledge-Intensive CBR (Aamodt, 2004), a specific type of CBR methodology that would allow agents to reason with semantic knowledge in addition to the syntactic properties of cases. A possible way to use KI-CBR to argue in open MAS is following an ontological approach to develop these kind of systems (Díaz-Agudo \& gonzález-Calero, 2007). In this way, ontologies can be used as case representation languages that integrate general terminological knowledge about CBR, argumentation and specific application domains. Further research to specify how to store arguments and dialogues in the cases of a KI-CBR system and how to reason with 
them must be carried out. Moreover, an intensive study to determine which type of ontology would be suitable for describing the argumentation concepts in the cases must be performed.

Another challenge that must be dealt with is how to communicate arguments between the agents of a particular MAS or between the agents of different systems. When working with open MAS, where the system dynamicity and the heterogeneity between agents is assumed by default, this functionality is particularly challenging. The research in this area has already been started by the ASPIC community, which is developing its standardisation proposal for an argument interchange format (AIF) (Chesñevar et al., 2006). The format introduces an abstract formalism for representing concepts about arguments, argument networks, communication and argumentation context in (closed and open) MAS capable of argumentation-based reasoning. Since the AIF is being agreed upon the argumentation and MAS expert research communities, it is likely to be adopted by many researchers as an standard for argument communication. Therefore, considering this proposal when deciding how to represent the syntax and semantics of the cases in a case-based argumentation framework would be advisable for preserving compatibility with other frameworks.

Finally, during the argumentation dialogue, agents can contract commitments that ensure mutually beneficial agreements. Commitment representation and management in different types of dialogues is still an open issue. We propose CBR as a way of coping with this challenge. For instance, when an agent is going to contract a new commitment, it could use CBR to check the similarity with the previously contracted commitments and ensure that the new commitment can be contracted (and will not compromise the case-base consistency).

Reasoning with commitments by using CBR could also be useful to determine, on the face of the final results obtained by the fulfilment of past commitments, if contracting again a similar commitment would be really profitable. Moreover, CBR could also be used to generate warrants about the agent's capability to fulfil a specific commitment by showing cases that represent similar commitments that were fulfilled by the same agent in the past.

\subsection{CBR Roles in the Argumentation Process}

\section{CBR-based Argument Generation, Selection and Evaluation}

Depending on the contents of the case-base, the CBR methodology can play different roles in the argumentation process. The most obvious role (and the one that appears in all frameworks analysed in this research) is to use the case-based reasoning cycle to perform the entire or part of the argument management process (i.e. generate, select and evaluate arguments). However, this process has been implemented by using a wide range of techniques without any standardisation in each current approach (see Table 1). Furthermore, the selection process in almost all frameworks (except for the AMAL framework) relies on domain-dependent rules and pre-established preference relations. This fact makes increasingly difficult to compare and evaluate the strengths and weaknesses of the CBR-based frameworks for argumentation in MAS.

Over the last years, explanation techniques that make the CBR solving process more understandable have gained an increasing interest (Sørmo, 2005). If case-based arguments are conceived as explanations that justify the position of an agent in a discussion, the explanation techniques developed in CBR systems could be a good alternative to standardise the generation, selection and evaluation of arguments in MAS. A preliminary work in this direction has been developed in the AMAL framework with the use of LID to generate justified predictions about the class of new problems, but it only applies to classification domains. Also, in the HERMES framework the case-base of the system is used to generate warrants that support the arguments asserted by the users. However, these cases only provide additional information that helps the user to select the best argument to instantiate and do not provide a formal explanation for the generation of specific arguments. Note that anyway, the user is who finally decides which argument poses at any step of the argumentation dialogue, no matter which is the recommendation provided by HERMES. In the rest of frameworks, the only explanation for generating particular arguments among the set of all possibilities is the similarity between the current and the previous argumentation dialogues. 
Other interesting application of explanations could be that the recipient agent uses them to check the correctness of the argument generation process when arguments are generated from the contents of the sender agent's case-base. In that way, these explanations could act as warrants for their associated arguments and ease the argument evaluation process for the recipient agent.

Besides that, if a knowledge intensive approach is used to store arguments (or pieces of information to generate arguments) in the agent's case-base, the implicit semantic knowledge of the relations between arguments could be also considered in the argument management process. From our point of view, there is a need for an intensive research that specifies how to apply explanation techniques for argument generation, selection and evaluation in different types of argumentation dialogues.

\section{CBR-based Argument Strategies}

As stated by Rawhan et al. (2003), bibliography about dialogue strategies in argumentation is hardly found. Therefore, other interesting role that the CBR methodology can play in argumentation processes in MAS is to generate argumentation strategies. Note that one of the main advantages of using CBR to manage argumentation in MAS is that it allows agents to learn from the process. In every framework studied in this research the underlying information about the current argumentation dialogue is partially stored in the form of cases when the process finishes. In addition, the agents of the AMAL framework can also learn during the argumentation dialogue by storing in their case-bases the cases that they receive from other agents. However, they do not learn how to predict the behaviour of particular agents, but only increase their own knowledge with the knowledge that other agents share with them.

CBR could also be used to learn agents' profiles and generate arguments to perform a strategic argumentation that would easily persuade specific types of agents. Some preliminary steps in this way have already been taken. The fist attempt to use CBR to provide information for building agents' profiles was performed in PERSUADER. In this framework the mediator agent uses the information about previous negotiation processes stored in the case-base to develop the behavioural model of an unknown agent and devise the best way to persuade it. Similarly, in Soh's framework the information of the cases is used to decide which type of arguments are best suited to convince an agent with a specific profile and to infer other parameters that influence the negotiation process (e.g. time constraints, resources usage, etc.). Nevertheless, in both cases the argumentation strategy is highly domain dependent and completely relies on previous knowledge. Although in PERSUADER the agents' models can be dynamically updated, the preference order that determines which argument must be ultimately posed depends on a pre-established hierarchy.

In a more dynamic and online way, the case-base could be used to store information about the agents' profile that could be gathered either by observing the current agents' behaviour, by learning information that the agents send during the dialogue or as a result of inquiry and information seeking processes. Therefore, this information could be used in the current argumentation process to generate and select better arguments to pose and to evaluate the incoming ones. Case-based argumentation strategies have to be further investigated and there is still much work to do in this area.

\section{CBR as Guide of the Interaction Protocol}

The interaction protocol between the agents of a MAS needs rules to govern the dialogue (e.g. the agents' entries and withdraws from the dialogue, the validity of proposals, the fulfilment of commitments and the termination of the dialogue). Our view is that CBR can play an important role as a useful tool to define such rules. For instance, the acceptable time to terminate an argumentation dialogue could be inferred from the information stored in the case-base about past similar arguments that ended in disagreement due to their excessive duration. On the other way round, the time to reach an agreement could be inferred from the time that took to reach similar agreements in the past.

When defining the current negotiation strategy, the Soh's framework already considers information about the time, number of steps and resources usage in previous negotiation processes. However, as pointed out before, the cases are completely situated and to be applicable in current negotiations they need to be adapted by using domain dependent adaption rules. In addition, only one case (the one that is 
potentially most similar to the current situation) is used to define the negotiation strategy. Our purpose is to develop algorithms that take into account the argumentation parameters of not only one specific case, but of a set of previous similar cases.

In addition, if CBR is used to generate and evaluate arguments, infinite dialogues can be avoided by introducing certain rules in the reasoning cycle. The AMAL framework, for example, does not allow an agent to generate the same case-based argument twice during a dialogue. This is a basic strategy, but still very effective. More elaborated rules could avoid circular arguments and prevent the introduction of fallacies that could deviate the argumentation dialogue from its main objective of reaching an agreement.

Finally, CBR could warrant the argumentation success by stopping the process or modifying certain parameters when the agents notice that the current dialogue is developing in a similar way to other precedent that found insuperable obstacles to reach a profitable agreement.

Note that the case-based guide of the interaction protocol could also be considered as another type of argumentation strategy and then, it should be included in the previous section. For clarity purposes, since this strategy is focused on the interaction process itself and not on the argumentation management, we have decided to study it in this separate section.

\subsection{Case-base Consistency Matters}

An important open issue that we have identified and that has received little attention in the literature is how to update arguments that were generated from past experiences to fit current argumentation dialogues. The case-base consistency is a critical issue to ensure the proper operation of a CBR system along the time. In the dynamic context of an open dialogue, where the agents can enter as new participants or well finish the interaction with the other agents, how a change of this type (environmental, in the agents' points of view, etc.) can affect to the validity of the case-base information must be considered. Otherwise, the arguments inferred from the case-base could become obsolete.

To ensure consistency, powerful algorithms to adapt and maintain the case-base must be implemented. Such algorithms must be able to adapt situated cases and make them applicable to the current problem or otherwise, eliminate them. Soh's model deals with this functionality to a certain extent, by using domainspecific adaption rules. However, due to the dynamism of the argumentation domain, such rules can quickly become obsolete. Therefore, the adaption methods must be as domain-independent as possible.

\subsection{Trust and Reputation}

None of the models that have been studied in this paper have taken reputation and trust issues into account as tools for preventing the system from unintentionally wrong or malicious behaviours. If the case-based argumentation framework is conceived to operate in open MAS these functionalities are essential. Before storing any information in the case-base during an argumentation dialogue between several agents, an agent must check the trustworthiness of such information. The opposite process is also necessary. If the profile that an agent has about other agent changes, resulting in a decrease of the the other's reputation, the information that comes from the interactions with this untrustworthy agent stored in the former's case base must be revised. Other important question that needs from further research is whether an agent must trust the information that it generates from its case-base by default. Trust and reputation issues must not be underestimated in argumentation frameworks in open MAS environments.

\section{Acknowledgements}

This work was partially supported by CONSOLIDER-INGENIO 2010 under grant CSD2007-00022 and by the Spanish government and FEDER funds under TIN2006-14630-C0301 project.

\section{References}

Aamodt, A. 2004, Knowledge-intensive case-based reasoning in Creek, in 7th European Conference on Case-Based Reasoning ECCBR-04, pp. 1-15. 
Aamodt, A. \& Plaza, E. 1994, Case-based reasoning: foundational issues, methodological variations and system approaches, AI Communications 7, no. 1, 39-59.

Aleven, V. \& Ashley, K. D. 1997, Teaching Case-Based Argumentation Through a Model and Examples, Empirical Evaluation of an Intelligent Learning Environment, in 8th World Conference of the Artificial Intelligence in Education Society, pp. 87-94.

Amgoud, L. 2003, A Formal Framework for Handling Conflicting Desires, in Symbolic and Quantitative Approaches to Reasoning with Uncertainty, Vol. 2711 of Lecture Notes in Computer Science, Springer Berlin: Heidelberg, pp. 552-563.

Amgoud, L. \& Kaci, S. 2004, On the generation of bipolar goals in argumentation-based negotiation, in 1st International Workshop on Argumentation in Multi-Agent Systems, ArgMAS, Vol. 3366 of Lecture Notes in Computer Science, Springer Verlag: Heidelberg, pp. 192-207.

Armengol, E. \& Plaza, E. 2001, Lazy induction of descriptions for relational case-based learning, in European Conference on Machine Learning, ECML-01, pp. 13-24.

Ashley, K. D. 1991, Reasoning with Cases and Hypotheticals in HYPO, International Journal of Man-Machine Studies 34, 753-796.

Atkinson, K. 2005, A dialogue game protocol for multi-agent argument over proposals for action, Autonomous Agents and Multi-Agent Systems. Special issue on Argumentation in Multi-Agent Systems 11(2), 153-171.

Aulinas, M., Tolchinsky, P., Turon, C., Poch, M. \& Cortés, U. 2007, Is my spill environmentally safe? Towards an Integrated Management of Wastewater in a river basin using agents that can argue, in 7th International IWA Symposium on Systems Analysis and Integrated Assessment in Water Management.

Bench-Capon, T. \& Dunne, P. 2007, Argumentation in Artificial Intelligence, Artificial Intelligence 171(10-15), 619938.

Branting, L. K. 1991, Building explanations from rules and structured cases, International Journal of Man-Machine Studies 34(6), 797-837.

Brüninghaus, S. \& Ashley, K. D. 2001, Improving the Representation of Legal Case Texts with Information Extraction Methods, in 7th International Conference on Artificial Intelligence and Law, ICAIL-01, pp. 42-51.

Brüninghaus, S. \& Ashley, K. D. 2003, Predicting the Outcome of Case-Based Legal Arguments, in 9th International Conference on Artificial Intelligence and Law, ICAIL-03, pp. 233-242.

Brüninghaus, S. \& Ashley, K. D. 2005, Generating Legal Arguments and Predictions from Case Texts, in 10th International Conference on Artificial Intelligence and Law, ICAIL-05, pp. 65-74.

Bylander, T. \& Chandrasekaran, B. 1988, Generic tasks in knowledge-based reasoning: The right level of abstraction for knowledge acquisition, Vol. 1, Academic Press, London, pp. 65-77.

Capobianco, M., Chesñevar, C. I. \& Simari, G. R. 2005, Argumentation and the Dynamics of Warranted Beliefs in Changing Environments, Autonomous Agents and Multi-Agent Systems 11(2), pp. 127-151.

Chesñevar, C., McGinnis, J., Modgil, S., Rahwan, I., Reed, C., Simari, G., South, M., Vreeswijk, G. \& Willmott, S. 2006, Towards an Argument Interchange Format, The Knowledge Engineering Review 21(4), pp. 293-316.

Daniels, J. J. \& Rissland, E. L. 1997, Finding legally relevant passages in case opinions, in 6th International Conference on Artificial Intelligence and Law, ICAIL-97, pp. 39-47.

Díaz-Agudo, B. \& González-Calero, P. A. 2007, Ontologies: A Handbook of Principles, Concepts and Applications in Information Systems, Springer, chapter: An Ontological Approach to Develop Knowledge Intensive CBR Systems, pp. 173-214.

Dung, P. M. 1995, On the acceptability of arguments and its fundamental role in nonmonotonic reasoning, logic programming, and N-person games, Artificial Intelligence 77, 321-357.

Fox, J. \& Parsons, S. 1998, Arguing about beliefs and actions, in Applications of Uncertainty Formalisms, Vol. 1455 of Lecture Notes in Computer Science, Springer Berlin: Heidelberg, pp. 266-302.

Gordon, T. F. \& Karacapilidis, N. 1997, The Zeno Argumentation Framework, in International Conference on Artificial Intelligence and Law, ICAIL-97, ACM Press, pp. 10-18.

Hamblin, C. L. 1970, Fallacies, Methuen, London.

Hulstijn, J. 2000, Dialogue Models for Inquiry and Transaction, University of Twente, The Netherlands.

Hulstijn, J. \& van der Torre, L. 2004, Combining Goal Generation and Planning in an Argumentation Framework, in Proceedings of the Workshop on Argument, Dialogue and Decision. International Workshop on Non-monotonic Reasoning, NMR-04.

Jakobovits, H. \& Vermeir, D. 1999, Dialectic Semantics for Argumentation Frameworks, in Proceedings of the 7th International Conference on Artificial Intelligence and Law, ICAIL-99, ACM Press, pp. 53-62.

Karacapilidis, N. \& Papadias, D. 2001, Computer supported argumentation and collaborative decision-making: the HERMES system, Information Systems 26(4), 259-277.

Karacapilidis, N., Trousse, B. \& Papadias, D. 1997, Using Case-Based Reasoning for Argumentation with Multiple Viewpoints, in 2nd International Conference on Case-Based Reasoning, ICCBR-97, pp. 541-552.

Karlins, M. \& Abelson, H. I. 1970, Persuasion: How Opinions and Attitudes are Changed, Springer.

MacKenzie, J. D. 1978, Question-begging in non-cumulative systems, Philosophical Logic 8(1), 117-133.

McBurney, P., Hitchcock, D. \& Parsons, S. 2007, The eightfold way of deliberation dialogue, International Journal of Intelligent Systems 22(1), 95-132. 
McBurney, P. \& Parsons, S. 2002, Dialogue Games in Multi-Agent Systems, Informal Logic. Special Issue on Applications of Argumentation in Computer Science 22(3), 257-274.

Modgil, S., Tolchinsky, P. \& Cortés, U. 2005, Towards formalising agent argumentation over the viability of human organs for transplantation, in 4th Mexican International Conference on Artificial Intelligence, MICAI05, pp. 928-938.

Ontañón, S. \& Plaza, E. 2006, Arguments and Counterexamples in Case-based Joint Deliberation, in AAMAS-06 workshop on Argumentation in Multi-Agent Systems, ArgMAS-06.

Ontañón, S. \& Plaza, E. 2007, Learning and Joint Deliberation through Argumentation in Multi-Agent Systems, in International Conference on Autonomous Agents and Multiagent Systems, AAMAS-07.

Ossowski, S., Julian, V., Bajo, J., Billhardt, H., Botti, V. \& Corchado, J. M. 2007, Open Issues in Open MAS: An Abstract architecture proposal, in Conferencia de la Asociacion Española para la Inteligencia Artificial, CAEPIA07, Vol. 2, pp. 151-160.

Parsons, S., Sierra, C. \& Jennings, N. R. 1998, Agents that reason and negotiate by arguing, Journal of Logic and Computation 8(3), 261-292.

Perelman, C. \& Olbrechts-Tyteca, L. 1969, The New Rhetoric: A Treatise on Argumentation, University of Notre Dame Press.

Prakken, H. \& Sartor, G. 1998, Modelling reasoning with precedents in a formal dialogue game, Artificial Intelligence and Law 6, 231-287.

Rahwan, I. 2006, Argumentation in Multi-Agent Systems, Autonomous Agents and Multiagent Systems, Guest Editorial 11(2), 115-125.

Rahwan, I. \& Amgoud, L. 2006, An Argumentation-based Approach for Practical Reasoning, in Proceedings of the 5th International Joint Conference on Autonomous Agents and Multiagent Systems, AAMAS-06, ACM Press, NY, USA, pp. 347-354.

Rahwan, I., Ramchurn, S. D., Jennings, N. R., McBurney, P., Parsons, S. \& Sonenberg, L. 2003, Argumentation-based negotiation, The Knowledge Engineering Review 18(4), 343-375.

Reed, C. \& Walton, D. 2005, Towards a Formal and Implemented Model of Argumentation Schemes in Agent Communication, in Proceedings of the 1st International Workshop in Multi-Agent Systems, ArgMAS-04.

Reiter, R. 1980, A logic for default reasoning, Artificial Intelligence 13, 81-132.

Rissland, E. L., Ashley, K. D. \& Branting, L. K. 2006, Case-based reasoning and law, The Knowledge Engineering Review 20(3), 293-298.

Rissland, E. L., Ashley, K. D. \& Loui, R. 2003, AI and Law: A fruitful synergy, Artificial Intelligence 150(1-2), 1-345.

Rissland, E. L., Skalak, D. B. \& Friedman, M. T. 1993, Bankxx: a program to generate argument through case-based search, in International Conference on Artificial Intelligence and Law, ICAIL-93, pp. 117-124.

Rissland, E. L. \& Skalak, D. B. 1991, CABARET: Rule Interpretation in a Hybrid Architecture, International Journal of Man-Machine Studies 34, 839-887.

Rittel, H. \& Webber, M. 1973, Dilemmas in a General Theory of Planning, Policy Sciences 4, 155-169.

Sadri, F., Toni, F. \& Torroni, P. 2001, Dialogues for Negotiation: Agent Varieties and Dialogue Sequences, in Proceedings of the 8th International Workshop on Agent Theories, Architectures, and Languages, ATAL-01, Vol. 2333 of Intelligent Agents VIII, Springer Berlin: Heidelberg, pp. 405-421.

Simari, G. R., García, A. J. \& Capobianco, M. 2004, Actions, Planning and Defeasible Reasoning, in Proceedings of the 10th International Workshop on Non-monotonic Reasoning, NMR-04.

Soh, L.-K. \& Tsatsoulis, C. 2001a, Agent-Based Argumentative Negotiations with Case-Based Reasoning, in AAAI Fall Symposium on Negotiation Methods for Autonomous Cooperative Systems, pp. 16-25.

Soh, L.-K. \& Tsatsoulis, C. 2001b, Reflective Negotiating Agents for Real-Time Multisensor Target Tracking, in International Joint Conference on Artificial Intelligence, IJCAI-01, pp. 1121-27.

Soh, L.-K. \& Tsatsoulis, C. 2005, A Real-Time Negotiation Model and a Multi-Agent Sensor Network Implementation, Autonomous Agents and Multi-Agent Systems 11(3), 215-271.

Sørmo, F., Cassens, J. \& Aamodt, A. 2005, Explanation in Case-Based Reasoning - Perspectives and Goals, Artificial Intelligence Review 24(2), 109-143.

Sycara, K. 1987, Resolving Adversarial Conflicts: An Approach Integrating Case-Based and Analytic Methods, $\mathrm{PhD}$ thesis, School of Information and Computer Science. Georgia Institute of Technology.

Sycara, K. 1989, Argumentation: Planning Other Agents' Plans, in 11th International Joint Conference on Artificial Intelligence, Vol. 1, Morgan Kaufmann Publishers Inc., pp. 517-523.

Sycara, K. 1990, Persuasive argumentation in negotiation, Theory and Decision 28, 203-242.

Tolchinsky, P., Modgil, S. \& Cortés, U. 2006a, Argument schemes and critical questions for heterogeneous agents to argue over the viability of a human organ, in AAAI Spring Symposium Series; Argumentation for Consumers of Healthcare.

Tolchinsky, P., Modgil, S., Cortés, U. \& Sànchez-Marrè, M. 2006b, CBR and Argument Schemes for Collaborative Decision Making, in Conference on Computational Models of Argument, COMMA-06, Vol. 144, IOS Press, pp. 71-82.

Tolchinsky, P., Cortés, U., Modgil, S., Caballero, F. \& López-Navidad, A. 2006c, Increasing Human-Organ Transplant Availability: Argumentation-Based Agent Deliberation, IEEE Intelligent Systems 21(6), 30-37. 
Tolchinsky, P., Atkinson, K., McBurney, P., Modgil, S. \& Cortés, U. 2007, Agents Deliberating Over Action Proposals Using the ProCLAIM Model, in 5th International Central and Eastern European Conference on Multi-Agent Systems, CEEMAS-07.

Toulmin, S. E. 1958, The Uses of Argument, Cambridge University Press.

van Eemeren, F. H. \& Grootendorst, R. 1984, Speech acts in argumentative discussions, Foris.

van Eemeren, F. H. \& Grootendorst, R. 2004, A Systematic Theory of Argumentation: The pragma-dialectical approach, Cambridge University Press.

Vázquez-Salceda, J., Cortés, U., Padget, J., López-Navidad, A. \& Caballero, F. 2003, The Organ Allocation Process: A Natural Extension of the Carrel Agent-Mediated Electronic Institution, AI Communications 16(3), 153-165.

Walton, D. 1996, Argumentation Schemes for Presumptive Reasoning, Studies in Argumentation Theory, Lawrence Erlbaum Associates.

Walton, D. \& Krabbe, E. C. W. 1995, Commitment in Dialogue: Basic Concepts of Interpersonal Reasoning, SUNY Press, Albany NY, USA.

Willmott, S., Vreeswijk, G., Chesñevar, C., South, M., McGinnis, J., Modgil, S., Rahwan, I., Reed, C. \& Simari, G. 2006, Towards an Argument Interchange Format for Multi-Agent Systems, in Proceedings of the AAMAS International Workshop on Argumentation in Multi-Agent Systems, ArgMAS-06, pp. 17-34. 